\title{
Degradation of Blue AlGaN/InGaN/GaN LEDs Subjected to High Current Pulses
}

\author{
Daniel L. Barton, Sandia National Laboratories, P. O. Box 5800, Albuquerque, NM 87185-1081 \\ Joachim Zeller, B. Scott Phillips, Pei-Chih Chiu, Sabrina Askar*, Dong-Seung Lee, Marek Osiński, \\ and Kevin J. Malloy, Center for High Technology Materials, The University of New Mexico, \\ Albuquerque, NM 87131-6081 \\ * on leave from Ecole Nationale Supérieure des Télécommunications, 46 rue Barrault, \\ F-75634, Paris Cédex 13, France
}

\begin{abstract}
Short-wavelength, visible-light emitting optoelectronic devices are needed for a wide range of commercial applications, including high-density optical data storage, full-color displays, and underwater communications. In 1994, high-brightness blue LEDs based on gallium nitride and related compounds (InGaN/AlGaN) were introduced by Nichia Chemical Industries[1]. The Nichia diodes are 100 times brighter than the previously available SiC blue LEDs. Group-III nitrides combine a wide, direct bandgap with refractory properties and high physical strength. So far, no studies of degradation of $\mathrm{GaN}$ based LEDs have been reported. Our study, reported in this paper, focuses on the performance of GaN LEDs under high electrical stress conditions. Our observations indicate that, in spite of a high defect density, which normally would have be fatal to other III-V devices, defects in group-III nitrides are not mobile even under high electrical stress. Defect tubes, however, can offer a preferential path for contact metals to electromigrate towards the $p-n$ junction, eventually resulting in a short. The proposed mechanism of GaN diode degradation raises concern for prospects of reliable lasers in the group-III nitrides grown on sapphire.
\end{abstract}

\section{INTRODUCTION}

The ability to produce a reliable, power- and cost-efficient blue light emitting diode has been of interest for some time to companies wanting to produce large, full color displays. Their eventual goal is to manufacture blue semiconductor lasers which can be combined with similar red and green devices to produce a laser projection video system. With enough power output, a system suitable for theaters is a possibility.

Because of the photon energies involved, blue light emission has, until recently, been unavailable. There are three primary choices of semiconductors for blue light emission. The use of silicon carbide $(\mathrm{SiC})$ has met with some success, but $\mathrm{SiC}$ is plagued by having an indirect band gap which makes photon emission inefficient. The group IIVI materials such as zinc selenide ( $\mathrm{ZnSe}$ ) are not yet mature enough for commercial production. The most promising materials are the group-III nitrides, with gallium nitride being the primary focus of research and development. The wurtzite polytypes of GaN, InN, and AIN can be deposited by various techniques with direct energy gaps ranging from $1.9 \mathrm{eV}$ for InN, to $3.4 \mathrm{eV}$ for $\mathrm{GaN}$, and $6.2 \mathrm{eV}$ for AlN. Alloys made from these materials can be tailored to a specific energy gap by creating ternary compounds.

There has been interest in these nitrides since the 1960's. Until recently, the growth of high quality (dislocation free) $\mathrm{GaN}$ with a low enough intrinsic hole concentration to allow for $p$-type doping has not been possible. Nichia Chemical Industries is the first company to produce high volumes of devices manufactured from $\mathrm{GaN}$ [1]. Based on past research, the best process to use, as demonstrated by Nichia, is to grow wurtzite GaN on (0001)c sapphire. Sapphire is widely available, stable at high processing temperatures, and easy to clean prior to epitaxial growth. The main disadvantage is that sapphire is not well lattice matched to GaN. $\alpha-\mathrm{Al}_{2} \mathrm{O}_{3}$ has hexagonal symmetry with lattice constants $\mathrm{a}=4.758 \AA$ and $\mathrm{c}=12.991 \AA$. The currently accepted numbers for stoichiometric GaN are $a=3.189 \AA$ and $c=5.185 \AA$. This mismatch usually results in the formation of double positioning boundaries, inversion domain boundaries, or dislocations at the $\mathrm{GaN} /$ sapphire interface [2]. Since there is also a thermal coefficient mismatch, strain develops in the GaN during post growth cooling. The growth of a thin, amorphous GaN layer or a thin $\mathrm{GaN}$ layer grown at a reduced temperature, reduces the strain and provides a platform for growing high quality GaN [3-13].

\section{BLUE LED HISTORY}

As early as 1991, Nichia had published work that demonstrated the operation of a blue light emitting diode 


\section{DISCLAIMER}

Portions of this document may be illegible in electronic image products. Images are produced from the best available original document. 
fabricated in GaN on sapphire [14]. The structure consisted of a simple $p-n$ junction and had a high forward turn-on voltage of about $5 \mathrm{~V}$. This was, however, the lowest reported forward turn-on voltage for any GaN device to date. This structure was soon followed by a double heterostructure diode with a silicon-doped InGaN layer ( $n$ type) to lower the forward turn-on and increase output efficiency $[15,16]$. Finally, in 1994, information on a double heterostructure device was published [1]. This LED used a co-doped zinc and silicon n-type InGaN layer sandwiched between two AlGaN layers (one $p$-type and one $n$-type) [17]. This reduced the forward turn-on voltage to its present level of about $3.6 \mathrm{~V}$. The structure is shown in figure 1 and is the subject for our analysis.

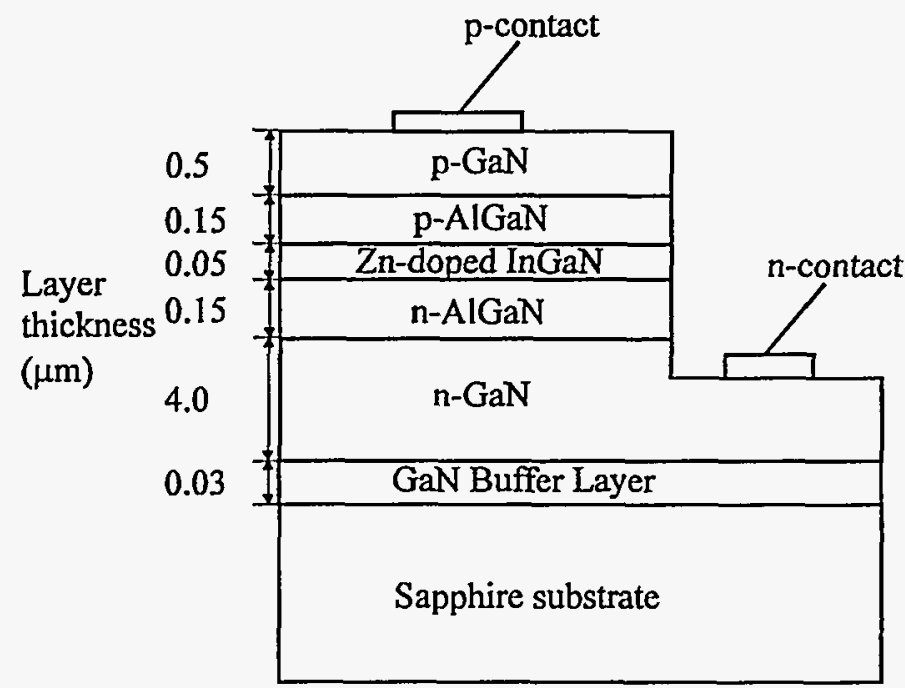

Figure 1 - Structure of AIGaN/InGaN/AIGaN DH blue LED [1].

\section{ELECTRICAL ANALYSIS OF SHORTED DEVICES}

The impetus for this paper was failures that occurred while output spectrum measurements were being made on these LEDs under high current conditions. The absolute maximum rating (as stated in the data sheet) for forward current are $30 \mathrm{~mA}$ for continuous wave (cw) and $100 \mathrm{~mA}$ for pulsed conditions. Under standard operating conditions, the diodes emit in a broad $(\sim 70 \mathrm{~nm})$ impurity-related band, centered at $450-460 \mathrm{~nm}$. The output power reaches a maximum at $\sim 75$ to $80 \mathrm{~mA}$ and then gradually decreases to about one-third of its peak value at $140 \mathrm{~mA}$ (the maximum forward current we have used for $\mathrm{cW}$ measurements). The impurity-related contribution peaks at $\sim 70 \mathrm{~mA}$ and then decreases due to heating. In contrast, the band-to-band component continues to increase beyond $70 \mathrm{~mA}$. This suggests that the current density, rather than temperature, is the main factor determining the efficiency of the band-to-band transitions, while elevated temperature suppresses the impurity-related transitions. This behavior is fully recoverable and no deterioration of device characteristics was observed during the measurements.

Similar changes in output spectra were observed under high-current pulsed conditions. Figure 2 shows the output spectra under pulsed conditions ( $100 \mathrm{~ns}$ rectangular pulses at $1 \mathrm{kHz}$ repetition rate) with amplitudes ranging from $40 \mathrm{~mA}$ to $1540 \mathrm{~mA}$. The band-edge emission around $380 \mathrm{~nm}$ increases at a faster rate than the impurity-related transitions around $460 \mathrm{~nm}$, and becomes dominant at very large current densities, while the impurity-related emission appeared to saturate.

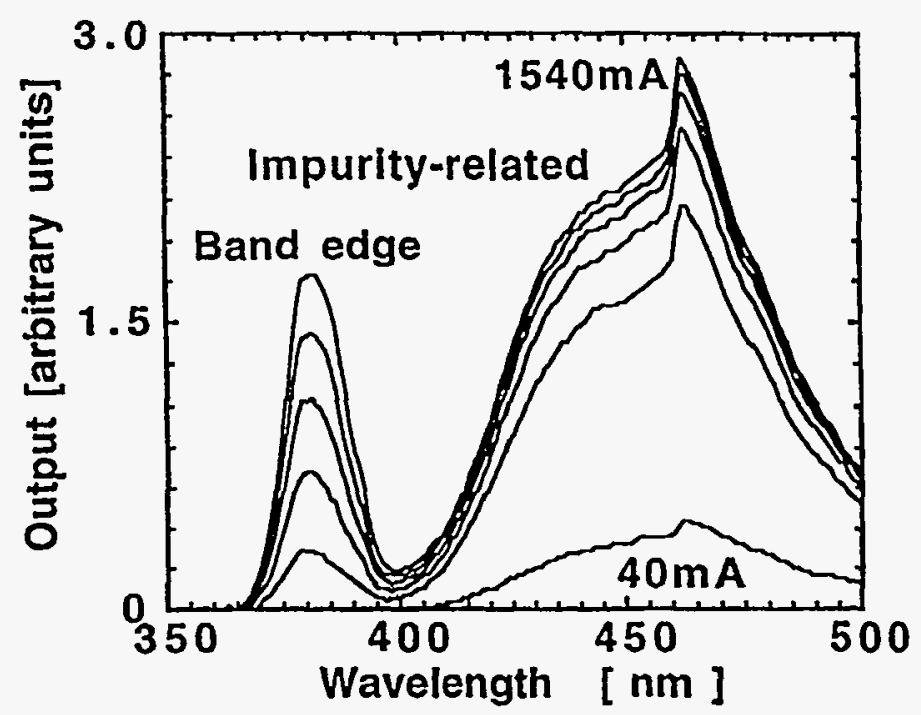

Figure 2 - Output spectra for various pulsed currents.

As a result of the high pulsed current measurements, several of the LEDs showed a degradation in I-V characteristics, some showed a low resistance ohmic short ( 40 to $800 \Omega$ ). The shorts were interesting in that they could be removed by applying a modest $(\sim 5 \mathrm{~V})$ reverse bias and re-created by applying a pulsed forward current. This process could be repeated so long as the LED remained encapsulated, i.e. the heat sink for the die was intact.

While the LED was in the shorted state, light output under $\mathrm{cw}$ conditions was not possible. The LED would still emit light under pulsed conditions. After a reverse bias was applied to remove the short, $\mathrm{cw}$ operation was once again possible. The effects of the stress were not completely removable, i.e. the LED could be placed in a shorted state or in a degraded state, not back into its original, unstressed state. The degraded state, not the shorted state was used for deep level transient spectroscopy (DLTS) and admittance spectroscopy measurements.

Figures 3 and 4 show I-V characteristics of both unstressed and stressed devices. The forward characteristics shown in figure 3 show both the I-V data for forward bias as well as the resistance (forward voltage divided by forward current). The I-V curves show a small difference, mostly a stress induced increase in conductivity at voltages below the 
threshold voltage $(\sim 2.8 \mathrm{~V})$. The resistance curves support this observation by showing a fairly constant resistance of about $10 \mathrm{k} \Omega$ for the degraded device versus several orders of magnitude greater resistance for the unstressed device. As expected, the difference between the two resistance curves is reduced after turn-on as the resistance across the junction will be very small compared to any other parallel resistance path. The reverse bias curves in figure 4 show a more dramatic difference. In this case, the normally small leakages found across reverse biased junctions are easily distinguishable from the ohmic path found across the stressed LED.

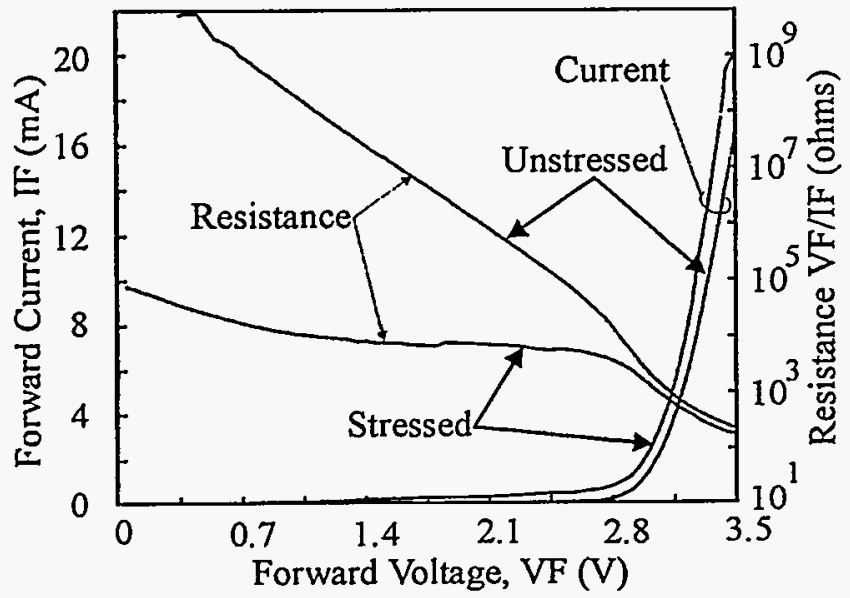

Figure 3 - Forward I-V characteristics and resistances of a stressed and an unstressed LED.

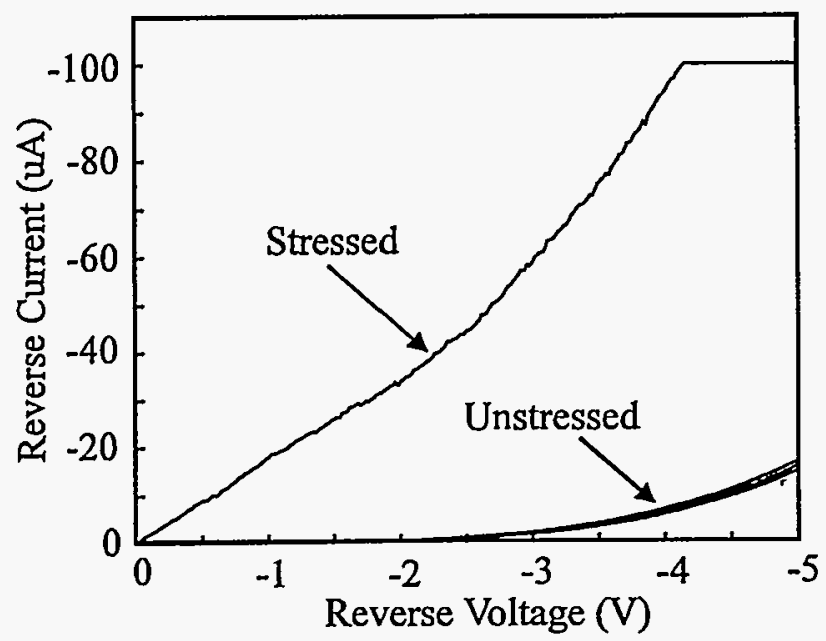

Figure 4 - Reverse I-V characteristics of one stressed and three unstressed LEDs.

In order to understand the physical phenomenon responsible for the changes in the characteristics of LEDs that have been subjected to high current stresses, several different tests were done.
DLTS was used to compare stressed samples with unstressed, control samples to see if the degradation was due to vacancies, interstitials, impurity atoms, etc. Figure 5 compares the DLTS spectrum from a stressed sample to an unstressed one. The DLTS measurements were made on a SULA Technologies spectrometer using a rate window of 50 ms. The system uses a MMR Technologies cooling stage capable of cooling down to $80 \mathrm{~K}$. Figure 5 shows that for the scanned temperature range, ( $\mathrm{T}>80 \mathrm{~K}$ ), there were no distinguishable peaks for either sample. The data suggests that there is a peak for temperatures less than $80 \mathrm{~K}$. The data does show that the low temperature peak would be indicative of the presence of shallow impurities and not from deep levels. The similarity between the stressed and unstressed samples suggests that the stress did not cause a significant change in any impurity level, especially deep level impurities.

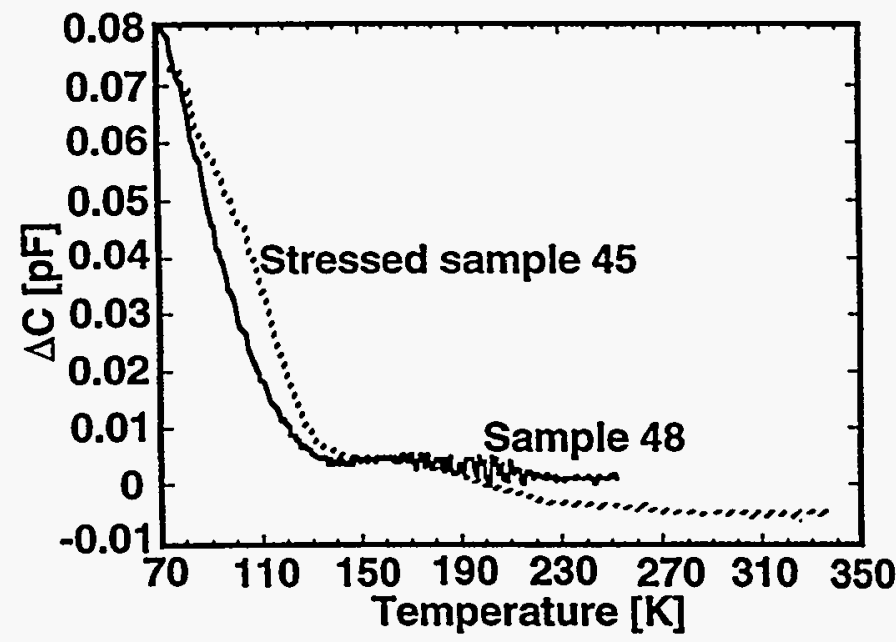

Figure 5 - DLTS spectra for stressed and unstressed LEDs.

This data contradicts recent reports on deep-level defects observed in $n$-type GaN $[18,19]$. The DLTS results on these LEDs show only an unresolved low temperature peak associated with minority carrier traps.

Admittance spectroscopy was done to determine if there was a detectable concentration of deep levels and, if so, what the activation levels of those levels were. These measurements were made using a Hewlett Packard 4192A impedance analyzer. The measurements were made at several different frequencies and temperatures using the same temperature control equipment as was used for the DLTS measurements. The measurements were made over a temperature range of $80 \mathrm{~K}$ to $340 \mathrm{~K}$ and a frequency range from $2 \mathrm{MHz}$ to $11 \mathrm{MHz}$.

Figure 6 shows a conductance versus temperature plot for an unstressed LED at several different frequencies. Figure 7 shows the same plot for a stressed LED. Both figures show that, in accordance with admittance spectroscopy theory, a peak could be found in the conductance and that the peak 
shifted to higher temperatures with higher frequencies to higher temperatures. From these data, an Arrhenious plot can be made whose slope gives the activation energy $\Delta \mathrm{E}=\mathrm{E}_{\mathrm{T}}-\mathrm{E}_{\mathrm{V}}$, where $\mathrm{E}_{\mathrm{T}}$ is the energy level of the trap and $\mathrm{E}_{\mathrm{V}}$ is the valence band energy. Figure 8 shows the Arrhenious plots for both the stressed and unstressed LEDs. From this figure, activation energies of $91 \mathrm{meV}$ for the stressed LED and $77 \mathrm{meV}$ for the unstressed LED are obtained. These results also indicate that no deep levels were detected, only shallow ones. The difference in activation energies is insignificant as the difference is within the standard deviation of the measurement on this system. These activation energies most likely correspond to the low temperature DLTS peak from figure 5 and are virtually unaffected by electrical stress.

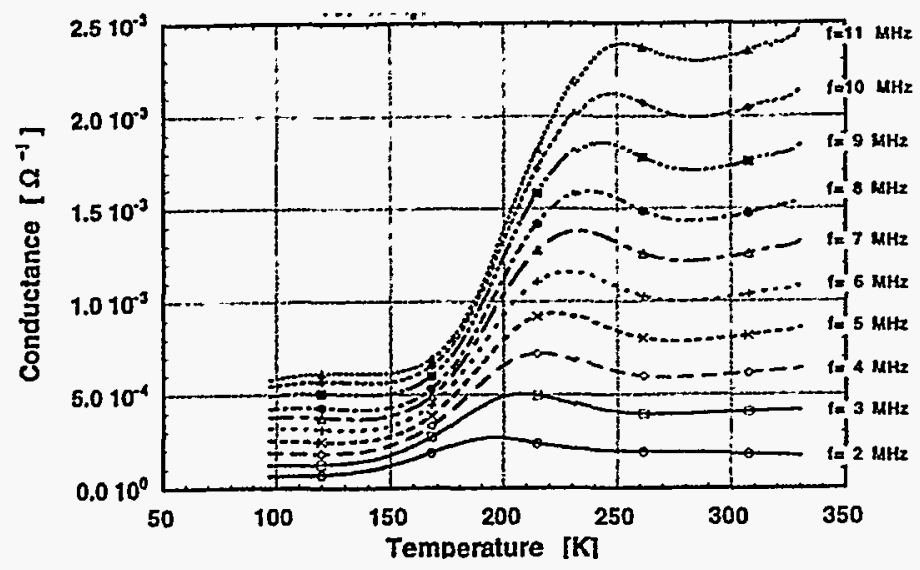

Figure 6 - Conductance versus temperature plot for an unstressed LED at several different frequencies.

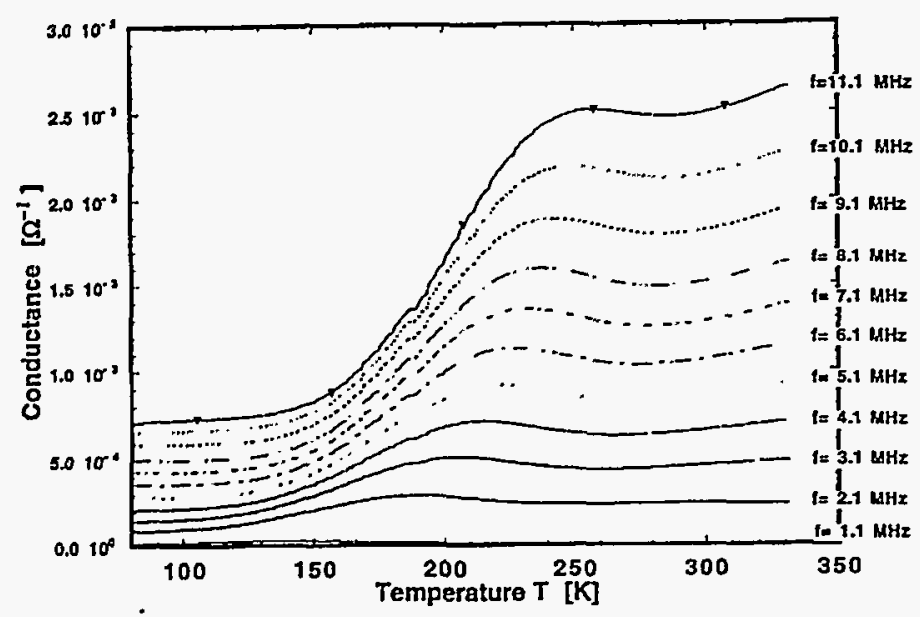

Figure 7 - Conductance versus temperature plot for a stressed LED at several different frequencies.

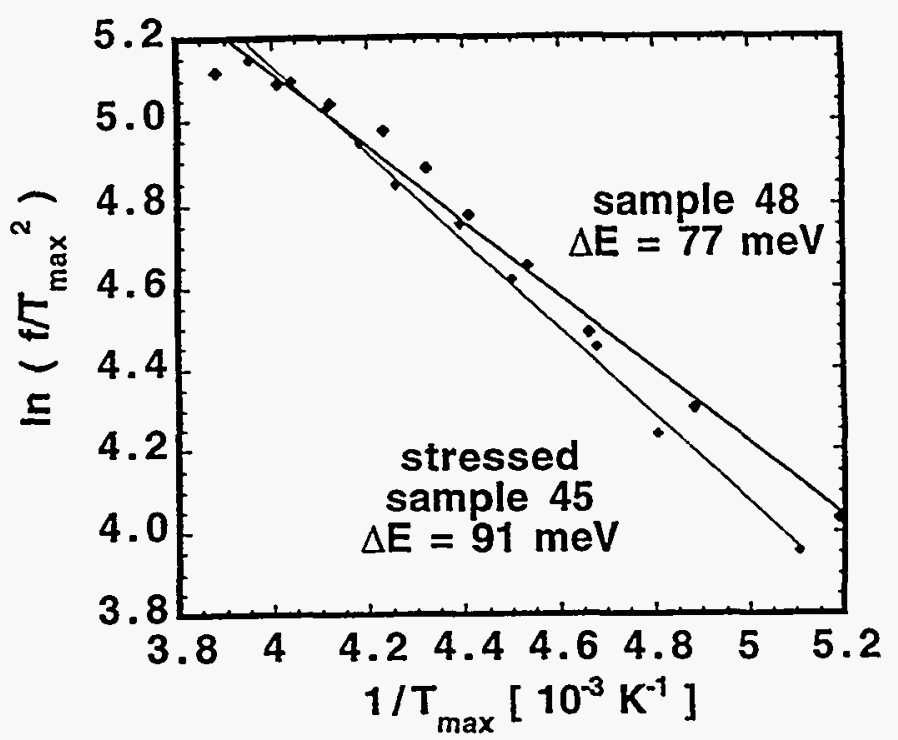

Figure 8 - Arrhenious plots from admittance spectroscopy data for both stressed and unstressed LEDs.

\section{EBIC ANALYSIS}

Electron beam induced current (EBIC) imaging was used to identify the location of low resistance path across the LED. Figures 9 is an EBIC image taken with a $7 \mathrm{keV}$ primary electron beam and a $10^{9}$ current gain. Figure 10 is a secondary electron image of the area imaged in figure 9. The EBIC image shows two distinct areas of increased current collection. The first is the edge of the $p$-contact area. Since this area allows the beam to come into contact with the junction, its presence in the EBIC images is expected. The only other signal is from the short across the junction. Figures 11 and 12 show higher magnification EBIC and secondary images, respectively, of the same defect region.

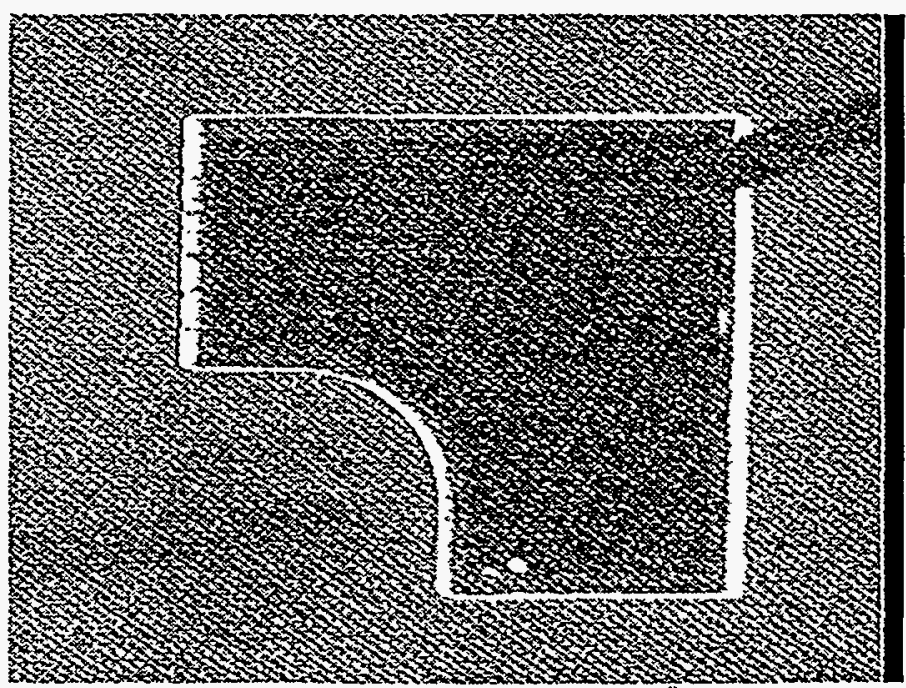

Figure 9 - EBIC image of stressed LED $\left(54 \mathrm{X}, 10^{9}\right.$ gain) at $7 \mathrm{keV}$. 


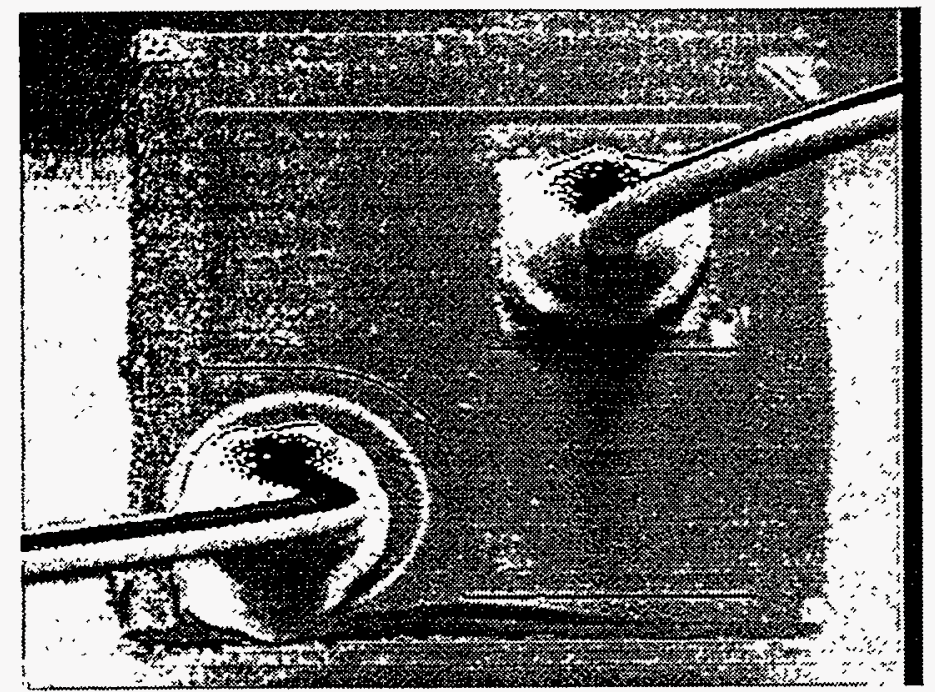

Figure 10 - Secondary electron image (54X) of area in figure 9.

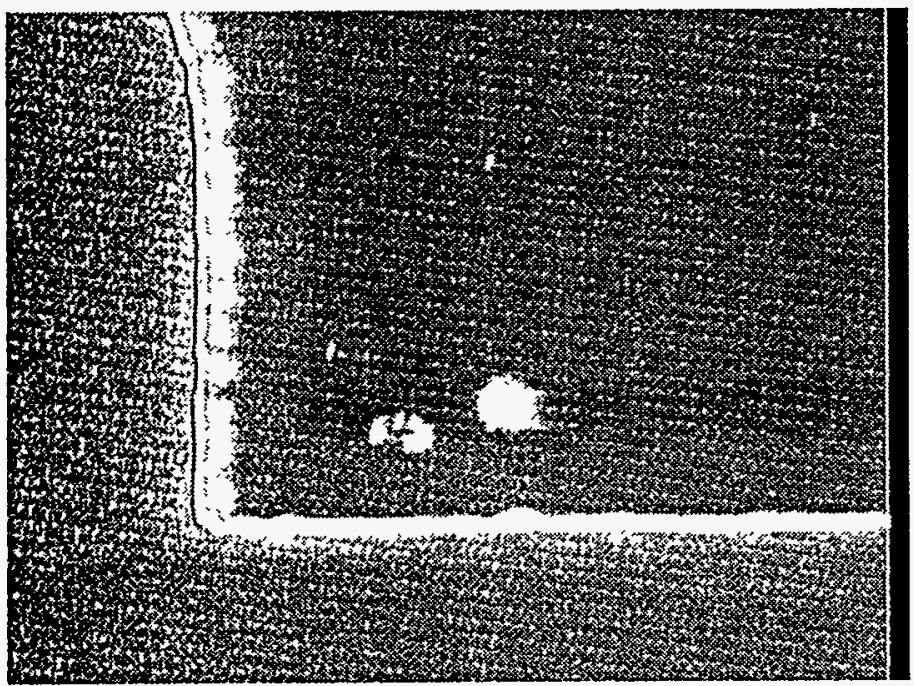

Figure 11 - Higher magnification EBIC image of stressed LED $\left(220 \mathrm{X}, 10^{9}\right.$ gain) at $7 \mathrm{keV}$.

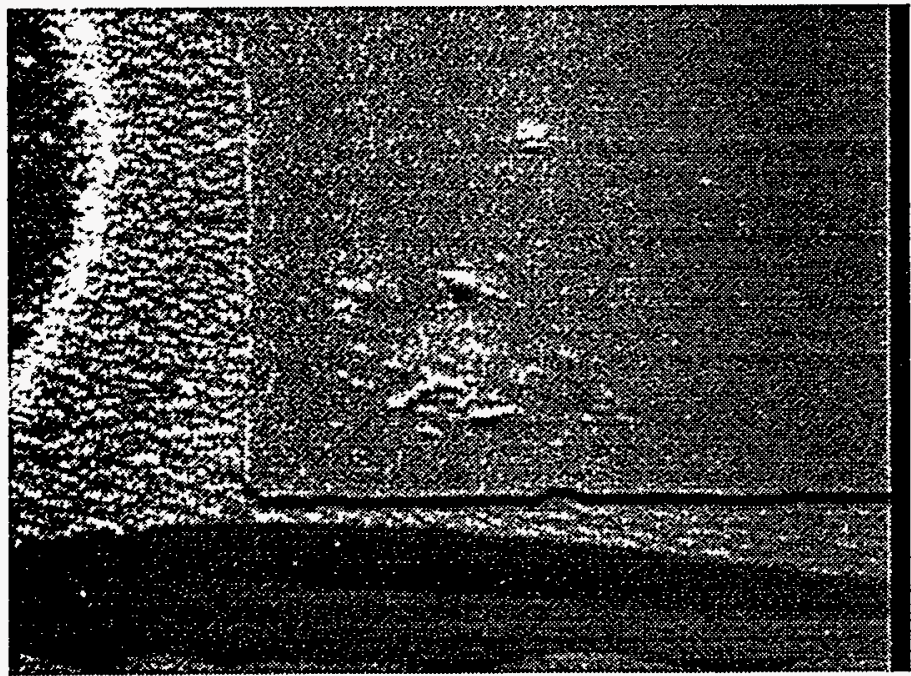

Figure 12 - Secondary electron image (220X) of area in figure 11.
Figure 13 shows a detail of the signal collected from the shorted region of the LED. This image shows that the high forward currents applied to this devices have caused metal from the $p$-contact to migrate across the junction. This figure also shows that the short consists of multiple metal fingers rather than a single thread. This observation supports the electrical data in that when the junction is reverse biased, the current would be carried almost entirely by the shorted area allowing these fingers to be fused open at relatively low voltages. Conversely, in forward bias, the junction would carry most of the current load.

Figure 14 is an EBIC image from the same LED using a $10 \mathrm{keV}$ beam. This image shows several dark regions caused by debris from the decapsulation process attenuating the electron beam as well as a strong signal from the entire junction area. At this energy, the electron beam penetrates deep enough to allow the entire $p-n$ junction area to collect the generated electron hole pairs. This energy does not, however, allow the location of the short to be imaged because of the strong signal from the $p-n$ junction. At 7 $\mathrm{keV}$, as shown in figures 9,11 , and 13 , the beam only penetrates deep enough to allow current collection in the vicinity of the short.

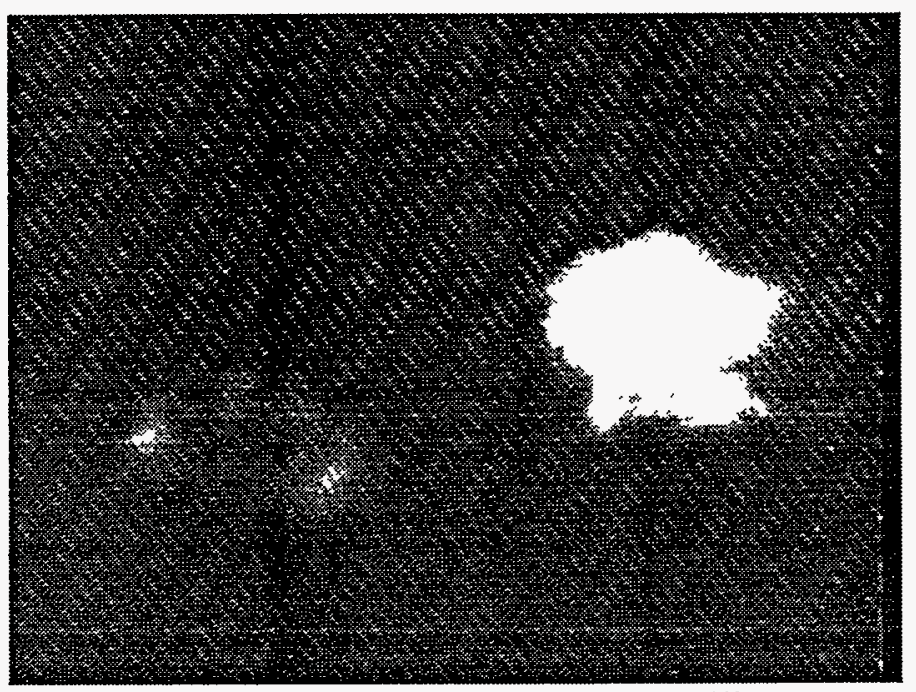

Figure 13 - EBIC image (905X) of stressed LED detailing shorted region.

\section{PHYSICAL IDENTIFICATION OF DEFECTS}

The LED shown in figures 9-14 was deprocessed to analyze the physical phenomenon causing the EBIC signal. Because of its small size and transparency, the LED had to be handled with care during the deprocessing operations. The gold $p$-contact was removed using aqua regia. Since the $p$-contact metallization is $\mathrm{Au} / \mathrm{Ni}$, the aqua regia successfully removed the gold and left a thin layer of nickel behind. Figure 15 shows two secondary images of the area identified 
using EBIC. The images clearly show that metal has migrated into the $\mathrm{GaN}$ under electrical stimulation.

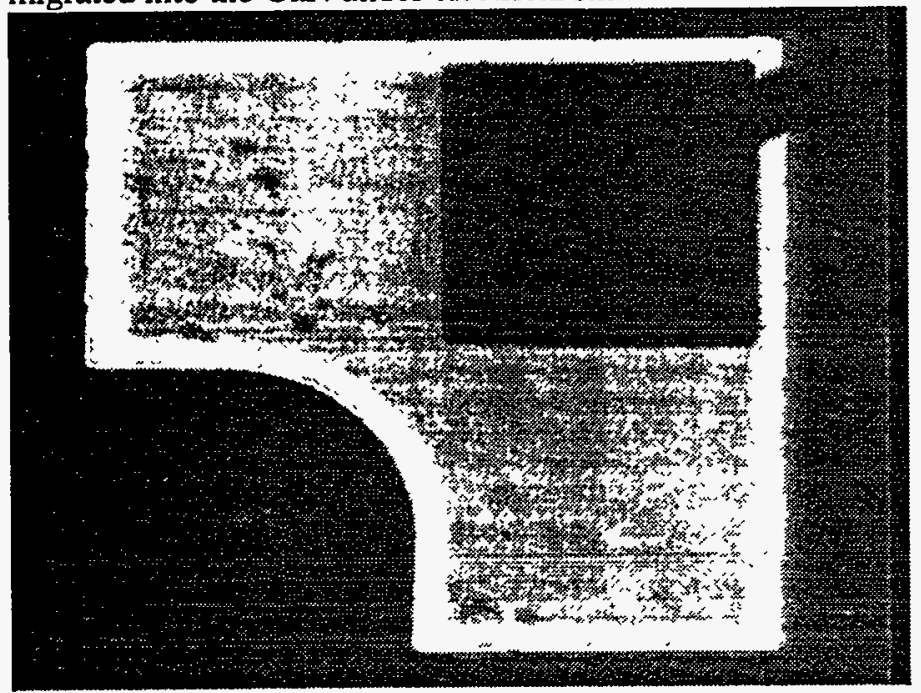

Figure 14 - EBIC image of stressed LED $\left(430 \mathrm{X}, 10^{9}\right.$ gain) at $10 \mathrm{keV}$.
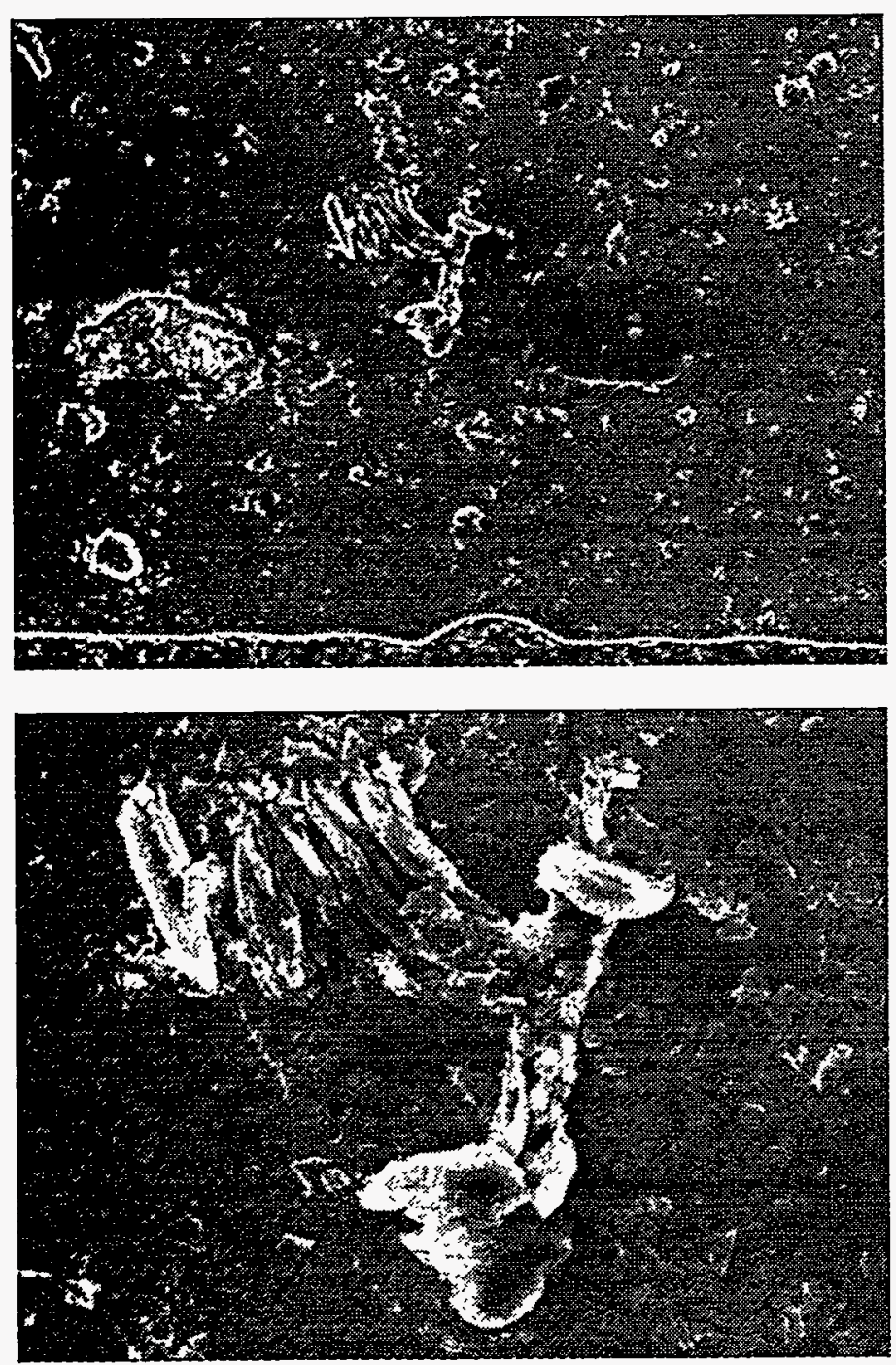

Figure 15-Secondary images of shorted area after $p$-contact removal. Top image is $2500 \mathrm{X}$, bottom image is $7500 \mathrm{X}$.

\section{TEM ANALYSIS}

As the main premise of this study was that the electromigration of contact metals was along crystalline defects or defect tubes, we needed to understand and quantify the defect density present in these devices. The best method to accomplish this was through the use of the transmission electron microscope (TEM). Since we only had single, packaged LEDs, we had to prepare tiny samples for cross section TEM analysis. The only way to accomplish this was with a focused ion beam system (FIB).

The main concerns with using a FIB for TEM sample preparation are the implantation of $\mathrm{Ga}$ into the sample and the creation of defects during the milling process. Even though the $\mathrm{Ga}$ beam is not scanned directly on the surface that will be studied in the TEM, enough Ga is implanted because of backscattered primary ions that its presence is observed. Several failure analysis facilities only use a FIB for TEM sample preparation when other, conventional methods are not feasible, which was the case for this work.

The first step in the sample preparation process was to mechanically thin the sample as much as possible to reduce the FIB milling time. After the LED was removed from its package, it was mounted on a polishing stub with wax. The sample was thinned on both sides (to allow examination of a specific region on the LED) using 600 grit sandpaper to a width of about $160 \mu \mathrm{m}$. The sliver was then transferred to a piece of scrap silicon that had been cut to a half-circle $3 \mathrm{~mm}$ in diameter and again held in place with wax. The silicon was to be the holder for the LED for both the FIB work and for analysis in the TEM.

The FIB work consisted of the deposition of a protective tungsten cap layer followed by three separate types of mills, all using a $30 \mathrm{keV}$ primary ion beam. The tungsten layer was approximately $5 \mu \mathrm{m}$ wide by $1 \mu \mathrm{m}$ thick and was deposited to protect the top surface of the TEM section.

The first, or rough mill was designed to remove as much of the GaN material in as short a time as possible. For this process, a large, $750 \mu \mathrm{m}$, aperture was used and a mill dose selected to cut to a depth of about $15 \mu \mathrm{m}$. Since we wanted to analyze a specific part of the LED, all three mills had to be done twice, once from each side. In all, an area of about $50 \mu \mathrm{m}$ by $160 \mu \mathrm{m}$ was milled to $15 \mu \mathrm{m}$ depth.

The second series of mills were performed with a $400 \mu \mathrm{m}$ aperture. This aperture allows more precise control of the mill process but slows the rate at which material is removed. The mills done in this phase were the full $50 \mu \mathrm{m}$ wide and $15 \mu \mathrm{m}$ deep, but only about $5 \mu \mathrm{m}$ long (with long referring to the dimension normal to the TEM sample area).

Finally, several regions were milled using a polish mill. For this, a $100 \mu \mathrm{m}$ aperture was selected to allow precise control of the beam as well as a finely polished surface. In 


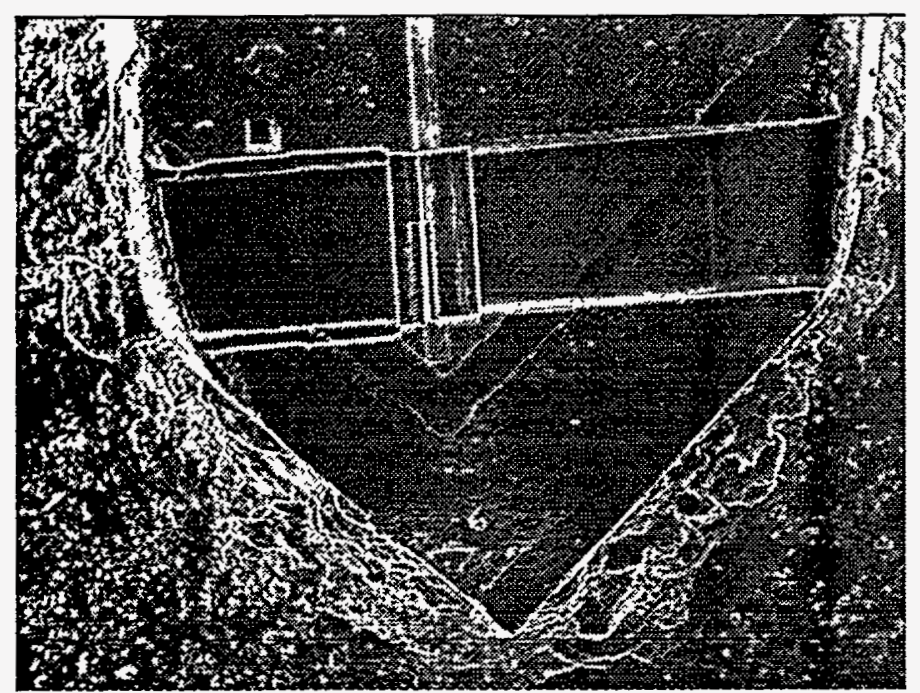

Figure 16 - SEM image (460X) of GaN LED after FIB milling.
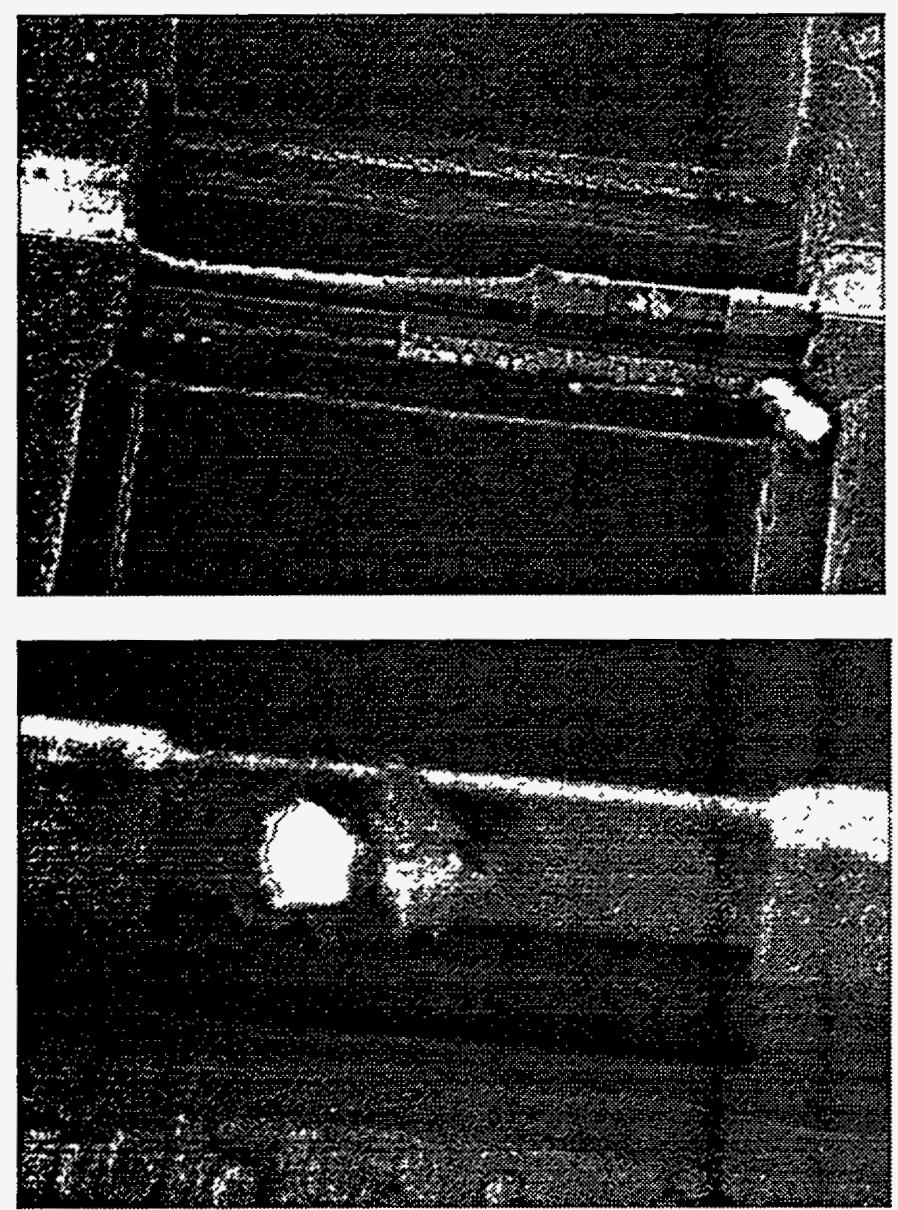

Figure 17 - SEM images of FIB sections: (top) after completion of three separate polish mills (2100X); (bottom) Close up of final FIB polish mill area (10400X).

order to avoid having to repeat the first two steps, three separate polish mills were made in two different sessions on the FIB. The first mill was made for a first look at the sample in the TEM. The second and third mills were performed later and shallower as the first polish mill eroded the tungsten cap, and about half of the GaN structure, which included the main area of interest.

Figure 16 is a SEM image of the overall milled area at low magnification. Figure 17 is a SEM image at 2100 times magnification of the three electron transparent regions that were analyzed in the TEM. These regions are estimated to be about $1000 \AA$ thick.

Figure 18 shows a TEM image of the defects threading through the GaN material. This image was taken with a two beam condition with $\mathbf{g}=(0331)$.

From this TEM analysis, we were able to calculate a defect density of $2.2 \times 10^{9} \mathrm{~cm}^{-2}$. The presence of the defect structure and the relatively high density of defects agrees with published data $[20,21]$ and with similar defect estimates based on inspection of the $n$-contact $\mathrm{GaN}$ material. Figure 19 shows an SEM micrograph of hexagonal shaped pits on the $\mathrm{GaN} n$-contact area. From this figure, a defect density of approximately $3 \times 10^{9} \mathrm{~cm}^{-2}$ is calculated.

These LEDs were manufactured using a process capable of growing high quality InGaN/AlGaN/GaN structures on sapphire [6]. The density of defects that we have measured does bring up the question of what defect density corresponds to high quality material. The GaN growth used is obviously good enough to achieve good yield of blue LEDs suitable for commercial sale.

\section{DISCUSSION}

Under normal operating conditions, Nichia diodes do not show any signs of rapid degradation. Lifetimes in excess of 10,000 hours are projected by the manufacturer.

The electromigration process is enhanced by the high voltage (tens of volts) required to drive high-current pulses through the device. Reversibility of the degradation of diode characteristics supports this interpretation.

Diode lasers will require very high current densities which may in turn cause electromigration. In order to prevent the latter from occurring, it is essential to design the lasers to operate at low voltages.

\section{CONCLUSIONS}

In this paper, we have shown that because of a relatively high intrinsic defect density, GaN based blue LEDs are susceptible to an electromigration type failure when subjected to high current pulses. The analysis relied on DLTS, output spectrum, and admittance spectroscopy measurements to prove that the degradation was not caused by the formation of deep levels or other charge trapping mechanisms. The data suggests that while these LEDs are not defect free, they are robust enough to withstand high levels of forward currents, many times the manufacturer's recommended limit, without catastrophic degradation. The 
presence of a large, $2.2 \times 10^{9} \mathrm{~cm}^{-2}$, density of defects decreases the resistance of the LED to electromigration type failure modes. Progress toward defect free $\mathrm{GaN}$ layer growth is necessary for the use of group-III nitrides in the manufacture of blue semiconductor lasers. Since these devices are normally operated at much higher current and photon densities, they will be more sensitive to the presence of crystalline defects.

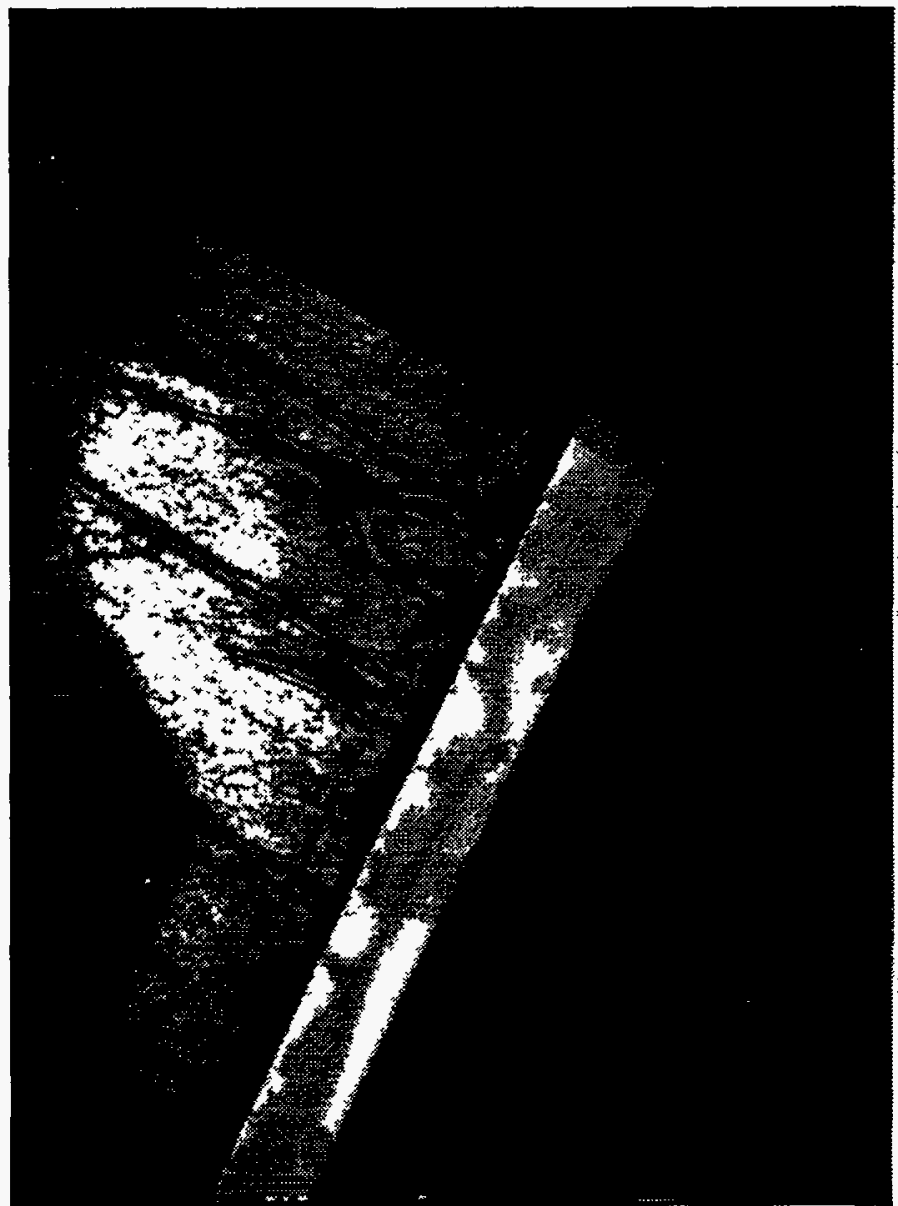

Figure 18 - TEM image of defects threading through GaN (21 $\mathrm{kX}$ at $200 \mathrm{keV}$ ).

\section{ACKNOWLEDGMENTS}

The authors would like to thank Ann Campbell and Jim Rife at Sandia National Laboratories for the preparation of the TEM sample using the focused ion beam system, Lumin Wang at the University of New Mexico for assistance with the TEM analysis, and Ann N. Campbell, Edward I. Cole, Jr., William M. Miller, Kenneth A. Peterson, and Paiboon Tangyungyong for reviewing this manuscript. This work was supported by ARPA, by the New Energy and Industrial Technology Development Organization (NEDO) of Japan and, at Sandia National Laboratories, by the U.S. Department of Energy under contract DE-AC04-94AL8500.

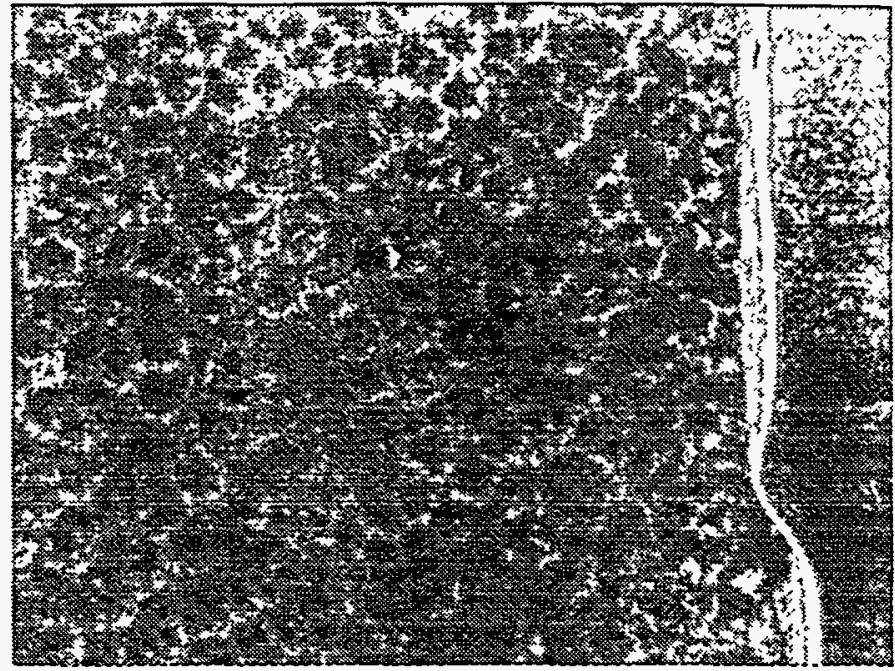

Figure 19 - SEM micrograph (5900X) of hexagonal pits in GaN n-contact layer.

\section{REFERENCES}

[1] S. Nakamura, T. Mukai, M. Senoh, "Candela-Class High-Brightness InGaN/AlGaN Double-Heterostructure Blue-Light-Emitting Diodes," Appl. Phys. Lett., Vol. 64, No. 13, 28 March 1994, p. 1687.

[2] S. Strite, and H. Morkoç, "GaN, AIN, and InN: A Review, J. Vac. Sci. Technol. B 10(4), Jul/Aug 1992, p. 1237.

[3] S. Strite, "GaN Core Relaxation Effects and Their Ramifications for P-Type Doping," Jap. J. Appl. Phys. Vol. 33, No. 5B, 15 May 1994, p. L699.

[4] S. Nakamura, N. Iwasa, and S. Nagahama, "Cd-Doped InGaN Films Grown on GaN Films," Jap. J. Appl. Phys., Vol. 32, No. 3A, 1 March 1993, p. L338.

[5] S. Nakamura, and T. Mukai, and M. Senoh, "Si-Doped InGaN Films Grown on GaN Films," Jap. J. Appl. Phys., Vol. 32, No. 1A/B, 15 January 1993, p. L16.

[6] S. Nakamura, and T. Mukai, "High-Quality InGaN Films Grown on GaN Films," Jap. J. Appl. Phys., Vol. 31, No. 10B, 15 October 1992, p. L1457.

[7] S. Nakamura, T. Mukai, M. Senoh, and N. Iwasa, "Thermal Annealing Effects on P-Type Mg-Doped GaN Films," Jap. J. Appl. Phys., Vol. 31, No. 2B, 15 February 1992, p. L139.

[8] S. Nakamura, M. Senoh, and T. Mukai, "Highly P. Typed Mg-Doped GaN Films Grown with GaN Buffer Layers," Jap. J. Appl. Phys., Vol. 30, No. 10A. October 1991, p. L1708.

[9] S. Nakamura. "GaN Growth Using GaN Buffer Layer," Jap. J. Appl. Phys., Vol. 30, No. 10A, October 1991, p. L1705. 
[10] S. Nakamura, , N. Iwasa, M. Senoh, and, T. Mukai, "Hole Compensation of P-Type GaN Films," Jap. J. Appl. Phys., Vol. 31, No. 5A, May 1992, p. 1258.

[11] S. Nakamura, Y. Harada, and M. Seno, "Novel Metalorganic Chemical Vapor Deposition System for GaN Growth," Appl. Phys. Lett., Vol. 58, No. 18, 6 May 1991, p. 2021.

[12] N. Yashimoto, T. Matsuoka, and A. Katsui, "Photoluminescence of InGaN Films Grown at High Temperature by Metalorganic Vapor Phase Epitaxy," Appl. Phys. Lett., Vol. 59, No. 18, 28 October 1991, p. 2251.

[13] S. Strite, J. Ruan, Z. Li, A. Salvador, H. Chen, D. Smith, W. Choyke, and H. Morkoç, "An Investigation of the Properties of Cubic GaN Grown on GaAs by Plasma-Assisted Molecular-Beam Epitaxy," J. Vac. Sci. Technol., Vol. 9, No. 4, July/August 1991, p. 1924.

[14] S. Nakamura, T. Mukai, and M. Senoh, "High-Power GaN P-N Junction Blue-Light-Emitting Diodes," Jap. J. Appl. Phys., Vol. 30, No. 12A, December 1991, p. L1998.

[15] S. Nakamura, M. Senoh, and T. Mukai, "P-GaN/NInGaN/N-GaN Double-Heterostructure Blue-LightEmitting Diodes," Jap. J. Appl. Phys., Vol. 32, No. 1A/B, January 1993, p. L8.

[16] S. Nakamura, M. Senoh, and T. Mukai, "High-Power InGaN/GaN Double-Heterostructure Violet Light Emitting Diodes," Appl. Phys. Lett., Vol. 62, No. 19, 10 May 1993, p. 2390.

[17] S. Nakamura, 2nd Workshop on Wide Bandgap Nitrides, St. Louis, MO., October 17-18, 1994.

[18] P. Hacke, T. Detchprohm, K. Hiramatsu, N. Sawaki, K. Tadatomo, and K. Miyake, "Analysis of Deep Devels in n-type GaN by Transient Capacitance Methods," $J$. Appl. Phys., Vol. 76, No. 1, 1 July 1994, p. 304.

[19] W. Gotz, N. Johnson, H. Amano, and I. Akasaki, "Deep Level Defects in n-type GaN," Appl. Phys. Lett., Vol. 65, No. 4, 25 July 1994, p. 463.

[20] T. Humphries, C. Sukow, R. Nemanich, J. Posthill, R. Rudder, S. Hattangady, and R. Markunas, "Micorstructural and Optical Characterization of GaN Films Grown by PECVD on (0001) Sapphire Substrates," Mat. Res. Soc. Symp. Proc. Vol. 162, 1990, p. 531.

[21] Z. Sitar, M. Paisley, and R. Davis, "Structural Defects in GaN Epilayers Grown by Gas Source Molecular Beam Epitaxy," Mat. Res. Soc. Symp. Proc. Vol. 162, 1990, p. 537. 